\title{
EFFECT OF RAYLEIGH NUMBER ON INTERNAL ECCENTRICITY IN A HEATED HORIZONTAL ELLIPTICAL CYLINDER TO ITS COAXIAL SQUARE ENCLOSURE
}

\author{
Abdelkrim BOURAS* \\ Department of Physics, Faculty of Sciences \\ University Mohamed Boudiaf of M'sila \\ M'sila, ALGERIA \\ Laboratory of Energetic Physics, University Frères Mentouri Constantine 1 \\ Constantine, ALGERIA \\ E-mail: karimbouras2006@yahoo.fr \\ Djedid TALOUB \\ Department of Physics, Faculty of Sciences \\ University Mohamed Boudiaf of M'sila \\ M'sila, ALGERIA \\ Laboratory of Renewable Energy and Sustainable Development (LRESD) \\ University Frères Mentouri Constantine1 \\ Constantine, ALGERIA \\ Zied DRISS \\ Laboratory of Electromechanical Systems (LASEM) \\ National School of Engineers of Sfax (ENIS), University of Sfax \\ Sfax, TUNISIA
}

\begin{abstract}
This paper deals with numerical investigation of a natural convective flow in a horizontal annular space between a heated square inner cylinder and a cold elliptical outer cylinder with a Newtonian fluid. Uniform temperatures are imposed along walls of the enclosure. The governing equations of the problem were solved numerically by the commercial code Fluent, based on the finite volume method and the Boussinesq approximation. The effects of Geometry Ratio GR and Rayleigh numbers on fluid flow and heat transfer performance are investigated. The Rayleigh number is varied from $10^{3}$ to $10^{6}$. Throughout the study the relevant results are presented in terms of isotherms, and streamlines. From the results, we found that the increase in the Geometry Ratio B leads to an increase of the heat transfer coefficient. The heat transfer rate in the annulus is translated in terms of the average Nusselt numbers along the enclosure's sides. Tecplot 7 program was used to plot the curves which cleared these relations and isotherms and streamlines which illustrate the behavior of air through the channel and its variation with other parameters. The results for the streamlines, isotherms, local and average Nusselt numbers average Nusselt numbers are compared with previous works and show good agreement.
\end{abstract}

Key words: natural convection, Newtonian fluid, geometry ratio, Rayleigh number, elliptical cylinder.

\section{Introduction}

Natural convection in a horizontal annulus kept at constant surface temperatures has been the subject of interest of many researchers due to its theoretical interest and its various engineering applications such as

\footnotetext{
${ }^{*}$ To whom correspondence should be addressed
} 
convection in buildings, fluid movement in solar energy collectors, cooling of electronic circuits, and cooling of nuclear reactors, heat exchangers. Kuehn and Goldstein [1]; [2] studied experimentally the effect of vertical eccentricity of the inner cylinder on the local and overall heat transfer coefficients. Their experimental data is commonly used to validate most of the recent numerical studies for Rayleigh numbers from $2.2 \times 10^{2}$ to $7.7 \times 10^{7}$. Manal et al. [3] investigated laminar mixed convection and radiation through an inclined rectangular duct with an interior circular tube numerically for a thermally and hydrodynamicaly fully developed flow. The two heat transfer mechanisms of convection and radiation are treated independently and simultaneously. Lacroix and Joyeux [4] conducted a numerical study of natural convection heat transfer from two horizontal heated cylinders confined to a rectangular enclosure having finite wall conductance. They indicated that wall heat conduction reduced the average temperature differences across the cavity, partially stabilized the flow and decreased natural convection heat transfer around the cylinders. Mohammed et al. [5] numerically studied the natural convection heat transfer in a twodimensional region formed by a constant heat flux horizontal flat tube concentrically located in a cooled horizontal cylinder. The model was solved using the FLUENT (computational fluid dynamics) package. The numerical simulations covered a range of hydraulic radius ratio equal to 5, 7.5, and 10 at orientation angles from $0^{\circ}$ up to $90^{\circ}$. Lee et al. [6] studied natural convection in enclosures with irregular walls and reviewed the schemes useful for numerical predictions. Cheng and Chao [7] investigated the buoyancy-driven flow in the annulus between two horizontal eccentric elliptical cylinders. Bouras et al. [8]; [9] investigated double diffusive natural convection in an annular space between two elliptical cylinders of confocal horizontal axes. They used the thermal Rayleigh numbers up to $5 \times 10^{5}$. In addition, they examined the influence of the Rayleigh number and Prandtl number on isotherms, isoconcentrations and streamlines. Li and Tong [10] studied natural convective heat transfer in the inclined rectangular cavities using three-dimensional numerical simulations and experimental measurements. Awasarmol and Pise, [11] experimentally investigated natural convection heat transfer enhancement of the perforated rectangular fin array at different angles of inclination. They observed that the perforation of fins enhances the heat transfer dissipation rates and the optimum perforation diameter depends on the inclination angle. El Shamy et al. [12] studied correlation for laminar natural convection between confocal horizontal elliptical cylinders. Bouras et al. [13] analyzed natural convective heat transfer from a heated horizontal elliptical cylinder to its coaxial square enclosure. Zhang et al. [14] analyzed natural convection heat transfer in a cylindrical envelope with an internal concentric cylinder with slots. Yu et al. [15] studied the effect of the Prandtl number on laminar natural convection heat transfer in a horizontal equilateral triangular cylinder with a coaxial circular cylinder. The Prandtl number was varied over a wide range from $10^{-2}$ to $10^{5}$.

Farooq et al. [16] studied the laminar natural convection inside a square enclosure containing different cross section inner pipes (circular, square and triangular) which have the same peripheral area with internal heat generation. Mehrizi et al. [17] carried out lattice Boltzmann simulation of natural convection in an elliptical-triangular annulus for different Rayleigh numbers $\mathrm{Ra}=10^{3}-10^{5}$. Xing et al. [18] numerically investigated natural convection in horizontal concentric annuli of varying inner shape where the inner and outer surfaces are kept at a constant temperature. The simulation was categorized into four groups based on the shape of the inner entity, which was either cylindrical, elliptical, square, or triangular. The present contribution presents a simulation of the problem as a mathematical model, which was solved numerically using the FLUENT-CFD. In this work we investigate the effect of the Rayleigh number and Geometry Ratio GR of the square heated on natural convection heat transfer.

\section{Description of the problem}

Figure 1 shows a cross section of the system. In this system, we consider the elliptical annular space characterized by the eccentricity of the outer elliptical tube $(e=0.7)$. The inner cylinder presents a heated square characterized by the dimension $h$. The outer cylinder presents a cold elliptic. The annular space is closed and contains an incompressible fluid with a kinematic viscosity $v$ and thermal diffusivity $\alpha$. The outer wall and the inner wall generate a vertical gradient in temperature (active walls), with Geometry Ratio GR $=L / b$. 


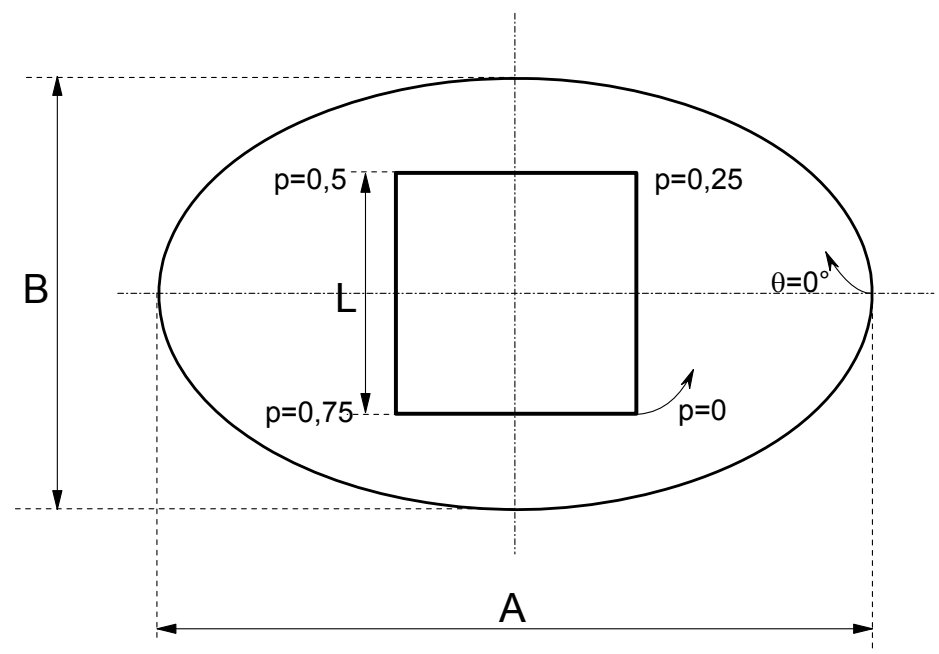

Fig.1. Schematic presentation of the physical model.

\section{Physical model and mathematical formulation}

\subsection{Simplifying hypotheses}

The flow of natural convection is caused by the thermal forces of pushes and remains laminar. It is supposed that the physical properties are constant except for the density of the mixture which depends on its temperature according to the relation of the approximation of Boussinesq

$$
\rho(T)=\rho_{0}\left[1-\beta_{t}\left(T-T_{0}\right)\right] .
$$

In these conditions, we admit that the problem is two-dimensional, permanent and laminar.

The characteristic quantities used to describe the problem are: the temperature differences $\Delta T=T_{H^{-}} T_{C}$ between the walls of the system and the thermal diffusivity $\alpha$ of the fluid. equation.

The mathematical model includes the continuity equation, the momentum equations and energy

The equation of continuity is written as follows

$$
\frac{\partial U}{\partial X}+\frac{\partial V}{\partial Y}=0
$$

The momentum equations are written as follows

$$
\begin{aligned}
& U \frac{\partial U}{\partial X}+V \frac{\partial U}{\partial Y}=-\frac{\partial P}{\partial X}+\frac{\partial^{2} U}{\partial X^{2}}+\frac{\partial^{2} U}{\partial Y^{2}} \\
& U \frac{\partial V}{\partial X}+V \frac{\partial V}{\partial Y}=-\frac{\partial P}{\partial Y}+\frac{\partial^{2} V}{\partial X^{2}}+\frac{\partial^{2} V}{\partial Y^{2}}+\frac{R a}{\operatorname{Pr}} \theta
\end{aligned}
$$

The equation of energy is written as follows 


$$
U \frac{\partial \theta}{\partial X}+V \frac{\partial \theta}{\partial Y}=\frac{1}{\operatorname{Pr}}\left(\frac{\partial^{2} \theta}{\partial X^{2}}+\frac{\partial^{2} \theta}{\partial Y^{2}}\right)
$$

The initial condition is

$$
\begin{aligned}
& U=V=0, \\
& \theta=\theta_{c}=\theta_{c} .
\end{aligned}
$$

In addition, the boundary conditions on the system are defined in the internal cylinder as follows

$$
\begin{aligned}
& U=V=0, \\
& \theta=\theta_{h} .
\end{aligned}
$$

However, in the external cylinder (ellipse), we can write

$$
\begin{aligned}
& U=V=0, \\
& \theta=\theta_{c} .
\end{aligned}
$$

The dimensionless parameters that govern the problem are the thermal Rayleigh $\left(\operatorname{Ra}_{t}\right)$, Prandtl number $(\mathrm{Pr})$ and Nusselt numbers $(\mathrm{Nu})$.

The thermal Rayleigh number for this problem is defined by

$$
R a_{t}=\frac{g \beta\left(T_{h}-T_{c}\right) b^{3}}{\alpha v}
$$

where $b$ is the characteristic length, which is chosen as the space between the two cylinders.

The number of Prandtl is defined as follows

$$
\operatorname{Pr}=\frac{v}{\alpha} .
$$

The local Nusselt number is defined on the characteristic length $L$ by

$$
\mathrm{Nu}_{L}=\left.L \frac{\partial \Phi}{\partial \boldsymbol{n}}\right|_{\text {wall }},
$$

here $\boldsymbol{n}$ is a normal vector on the wall and $\phi$ is the dimensionless temperature. The perimeter of the ellipse Pe and the perimeter of the square $P$ were chosen as the characteristic length $L$.

The average Nusselt number for the ellipse and the square can be evaluated by

$$
\overline{\mathrm{Nu}}_{\text {outer }}=\frac{1}{\mathrm{Pe}} \int_{0}^{\mathrm{Pe}} \mathrm{Nu}_{L} d \mathrm{Pe},
$$




$$
\overline{\mathrm{Nu}}_{\text {inner }}=\frac{1}{P} \int_{0}^{P} \mathrm{Nu}_{L} d P .
$$

The average Nusselt number for both surfaces is

$$
\mathrm{Nu}_{A V G}=\frac{\overline{\mathrm{Nu}}_{\text {outer }}+\overline{\mathrm{Nu}}_{\text {inner }}}{2} .
$$

\subsection{Meshing choice}

A typical grid distribution with uniform and orthogonal distribution is presented in Fig.2. It consists of 230x130 nodes.

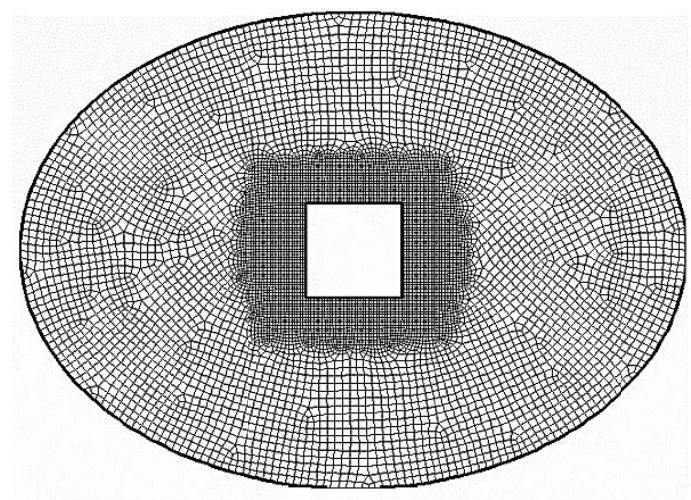

Fig.2. Grid distribution.

In this study, several grids were used arbitrarily for the considered configuration for $\mathrm{Ra}=10^{5}$ to observe their effect on the results. Table 1 shows, therefore, the variation of the average Nusselt number, according to the number of nodes. According to these simulations, the grid 230 X 130 appears more adequate to be considered since the relative error is weak.

Table 1. Variation of the average Nusselt number accordingto the number of nodes for $\mathrm{Ra}=10^{5}$.

\begin{tabular}{|l|l|l|l|l|l|l|}
\hline Mesh size & $180 \times 80$ & $200 \times 100$ & $210 \times 110$ & $220 \times 120$ & $230 \times 130$ & $240 \times 140$ \\
\hline $\mathrm{Nu}$ (avg) & 5.8826 & 5.6496 & 5.5818 & 5.4955 & 5.4319 & 5.4250 \\
\hline $\begin{array}{l}\text { Relative } \\
\text { Error (\%) }\end{array}$ & 4.1242 & 1.2147 & 1.5704 & & & \\
\hline
\end{tabular}

\section{Results and discussion}

The effect of different Rayleigh numbers on the natural convection of the heat transfer in an enclosure delimited by two horizontal confocal cylinders elliptic and square, filled of air $(\operatorname{Pr}=0.71)$ was studied for four different Rayleigh numbers. Results are presented in the form of streamlines, isotherms, local and average Nusselt numbers. The natural convection between the horizontal elliptic cylinders confocal by El Shamy et al. [12] was chosen for the validation of this study. These results were presented in the form 
of isotherms and of streamlines for two different Rayleigh numbers, as shown in Fig.3. Moreover, the local Nusselt number was compared with the results of El Shamy et al. [12] for different Rayleigh numbers extending from $10^{4}$ to $10^{5}$ (Fig.4). In the case of two elliptic horizontal confocal, the eccentricity of the inner and outer wall are taken 0.9 and 0.4 , respectively, and the Rayleigh number equal to $10^{4}$ (Fig.2). The local Nusselt numbers of the inner and outer cylindrical ellipse for two Rayleigh numbers are plotted in Fig.4. The result indicates an acceptable agreement with the results which are presented by El Shamy et al. [12]. In every case, the results show that two symmetrical cells of recirculation are formed on the right and left of the vertical symmetry of the cavity. It is due to the strength of buoyancy produced by the gradient of temperature. In these conditions, the fluid is raised on the side of the inner cylinder, and the flow becomes fresh and denser by the outer cylinder.

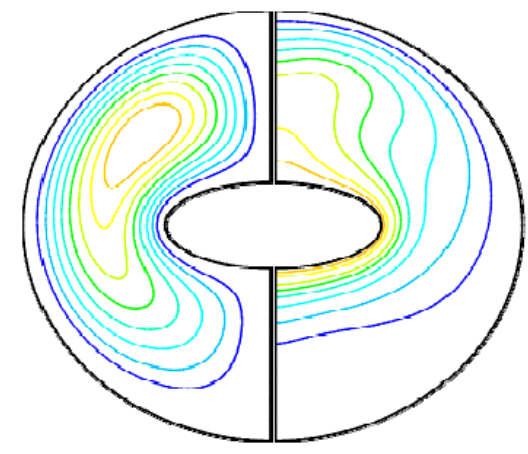

(a) Present study

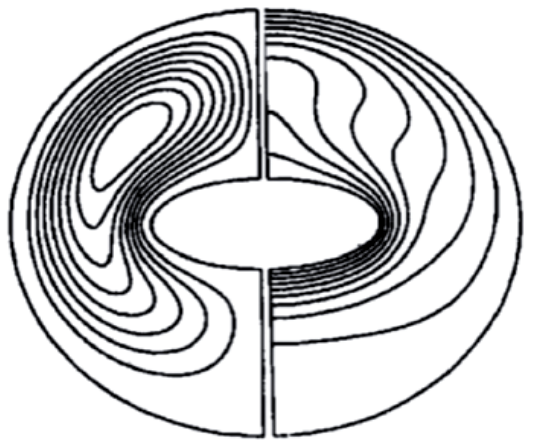

(b) El Shamy et al. [12]

Fig.3. Streamline (left half) and isotherms (right half) at $\mathrm{Ra}=10^{4}$.
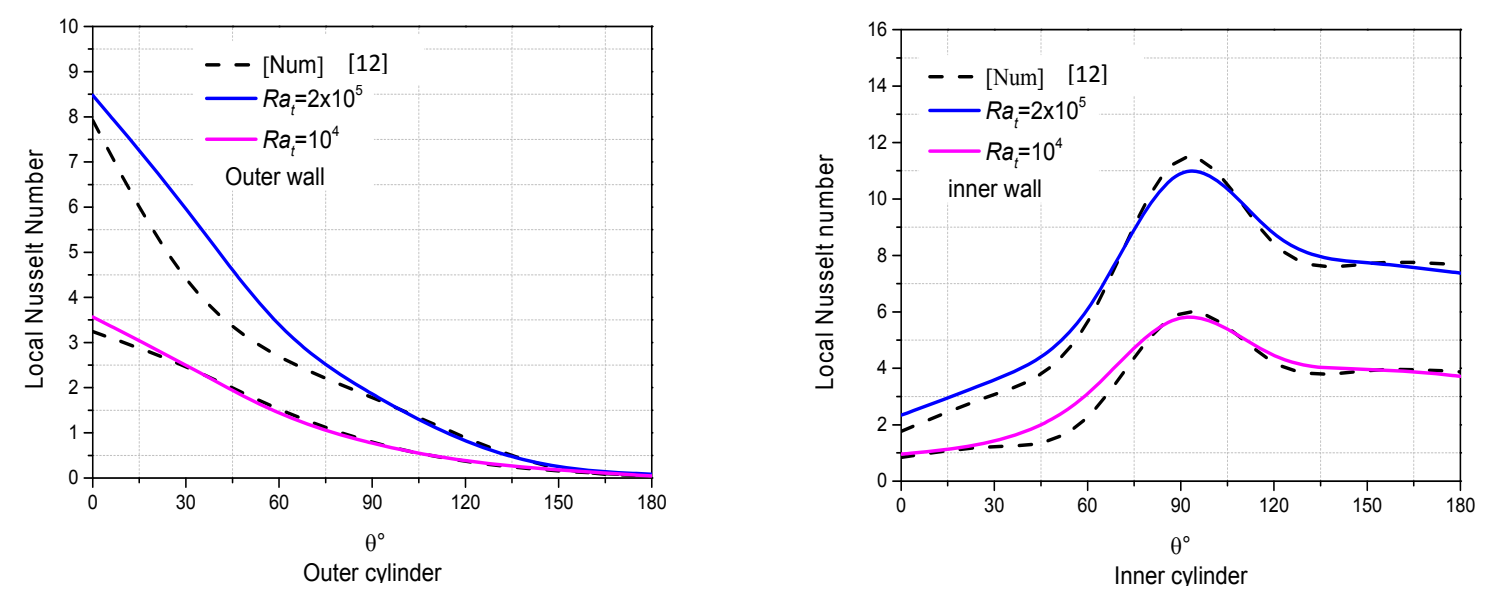

Fig. 4. Comparison of the local Nusselt number along the inner and outer ellipses with previous results [12].

\subsection{Effect of thermal Rayleigh number}

Figures 5, 6, 7 and 8 show the current lines and the isotherms distribution for different values of the Geometry Ratio GR and $\mathrm{Ra}_{\mathrm{t}}$. From these results, it can be seen that the flow is symmetrical with respect to the median vertical axis passing through the center of the inner square cylinder. The flow over the square cylinder creates a recirculation zone on both sides of the cavity for all values of GR and $\mathrm{Ra}_{\mathrm{t}}$. The flow is organized into two main counter-rotating cells. The cell on the left rotates in the counter clockwise direction while the right cell rotates clockwise. This is due to the displacement of the particles of the fluid towards the top of the chamber under the effect of buoyancy, by heating up along the hot wall. Then they move towards the bottom of the enclosure under the effect of gravity by cooling along the cold wall. For $\mathrm{Ra}_{\mathrm{t}}=10^{3}$, laminar 
convection is weak, the values of the current function are very low and almost similar. In this case, the heat transfer is mainly by conduction. For $\mathrm{Ra}_{\mathrm{t}}=10^{4}$, with $\mathrm{GR}=0.8$, we are always in a pseudo-conductive regime. The thermal transfers are therefore essentially conductive. The values of the current function appearing on these different figures are always small, convection is relatively weak. But with a Geometry Ratio GR different to GR $=0.8$, the isothermal lines change and eventually adopt the shape of a mushroom. The values of the current functions increase which means that convection intensifies. For $\mathrm{Ra}_{\mathrm{t}}=10^{5}$ and $\mathrm{Ra}=$ $10^{6}$, the increase in the thermal Rayleigh number presents in an intensification of natural convection.

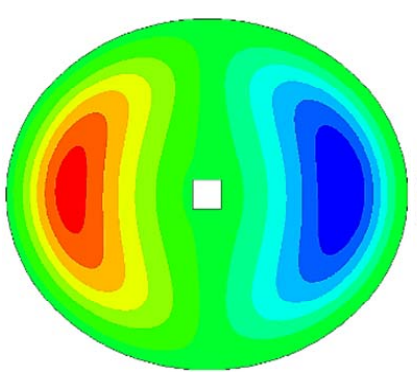

(a) $\mathrm{GR}=0.2$

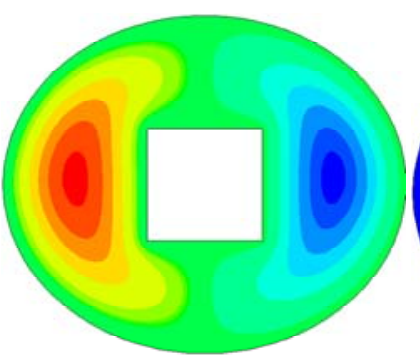

c) $\mathrm{GR}=0.6$
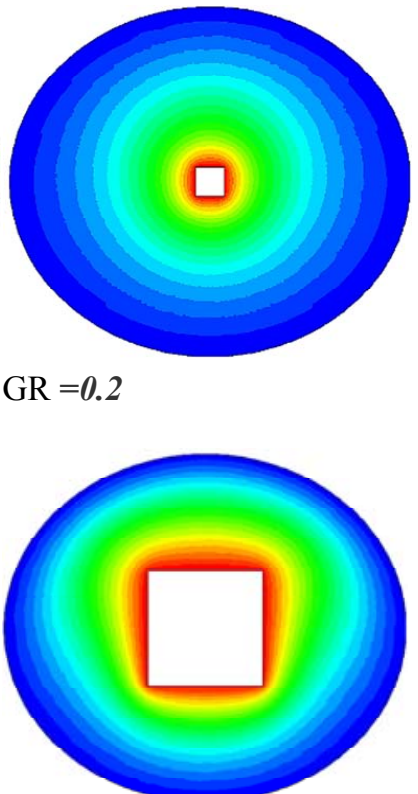

Fig.5. Current lines and isotherm distribution for $\mathrm{Ra}_{\mathrm{t}}=10^{3}$.
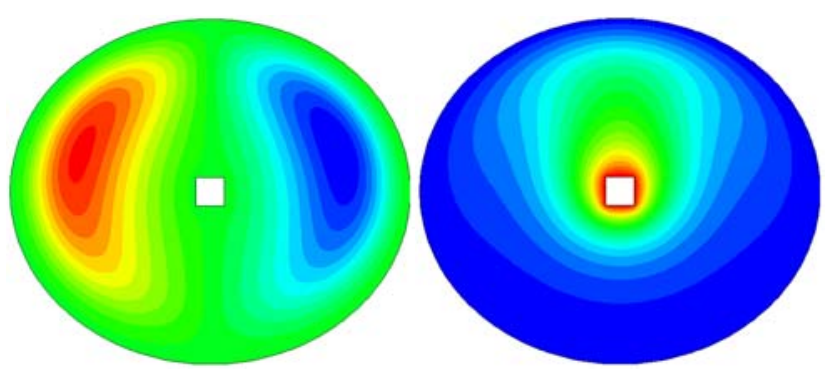

a) $\mathrm{GR}=0.2$
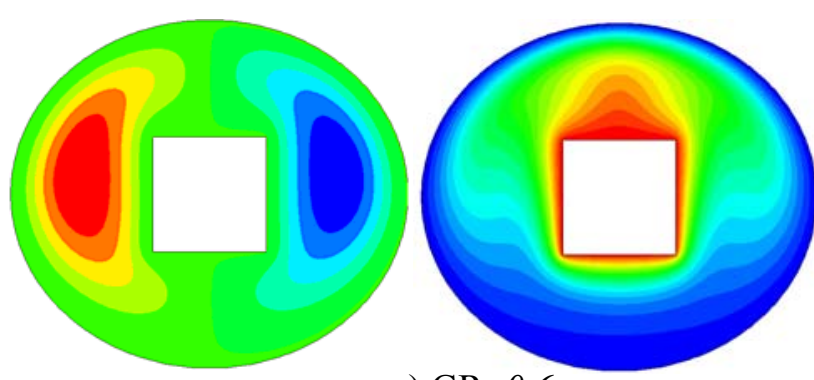

c) $\mathrm{GR}=0.6$
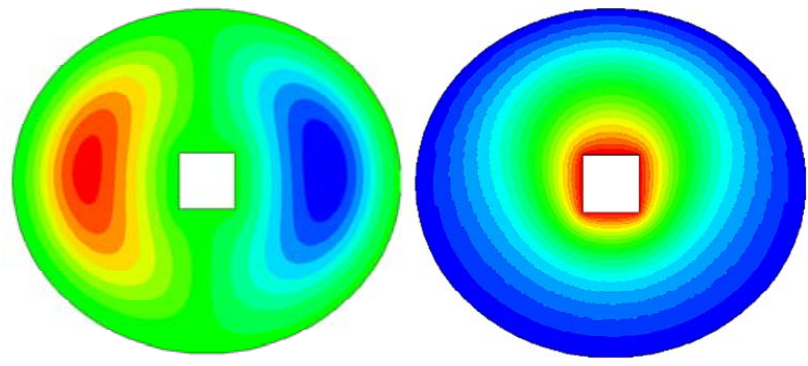

b) $\mathrm{GR}=0.4$
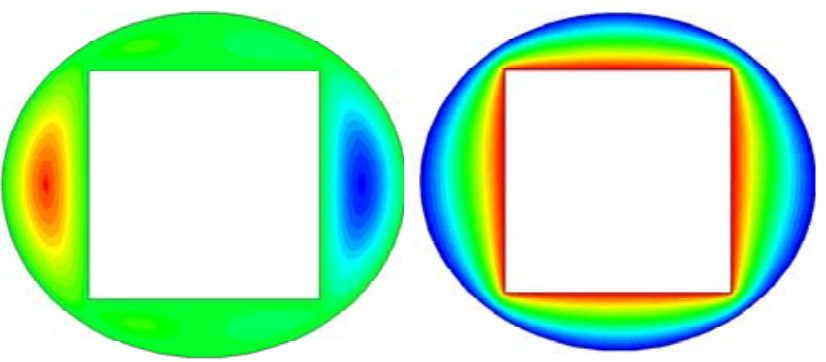

d) $\mathrm{GR}=0.8$

Fig.6. Current lines and isotherm distribution for $\mathrm{Ra}_{\mathrm{t}}=10^{4}$. 

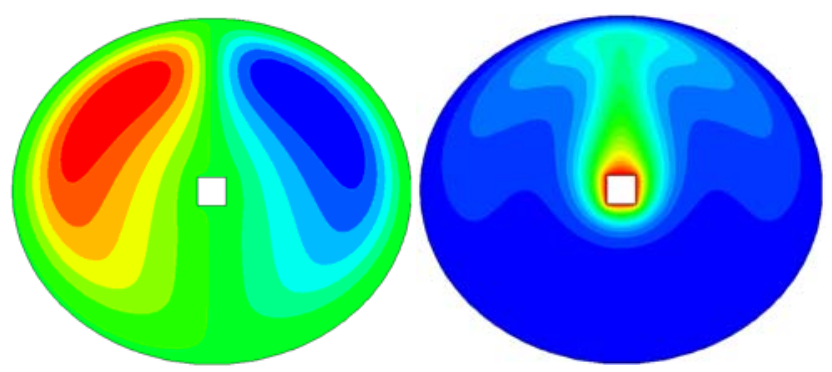

a) $\mathrm{GR}=0.2$

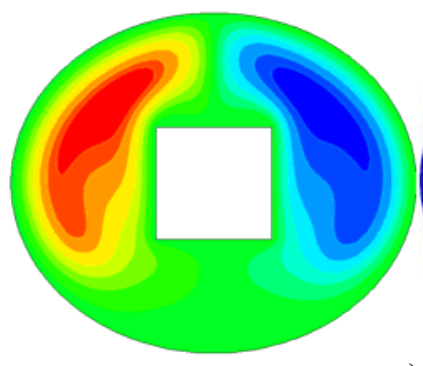

c) $\mathrm{GR}=0.6$

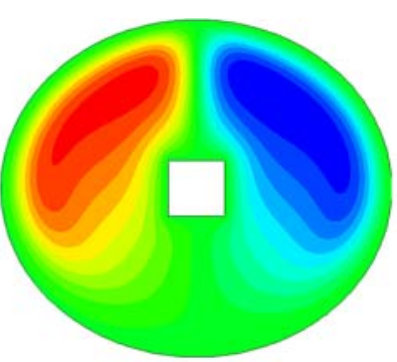

b) $\mathrm{GR}=0.4$

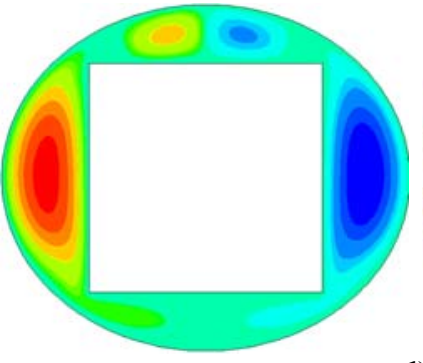

d) $\mathrm{GR}=0.8$
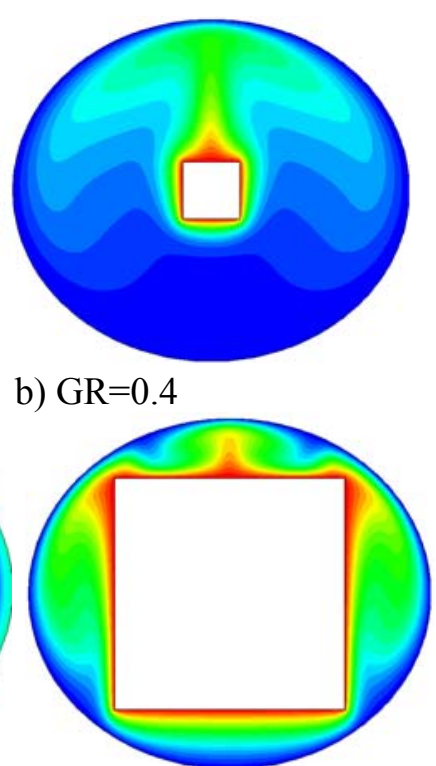

Fig.7. Current lines and isotherm distribution for $\mathrm{Ra}_{\mathrm{t}}=10^{5}$.
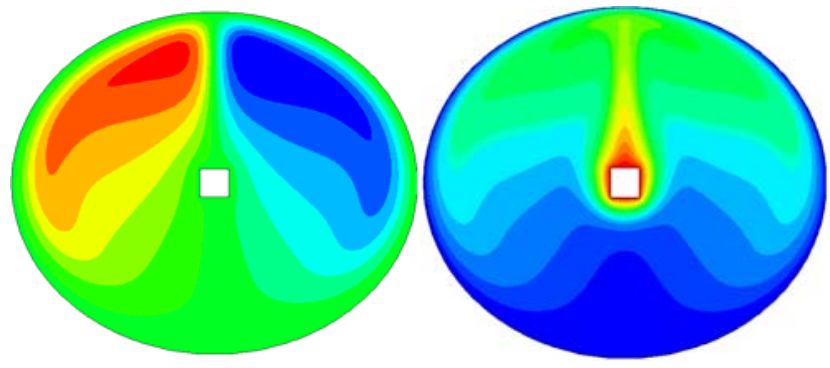

a) $\mathrm{GR}=0.2$

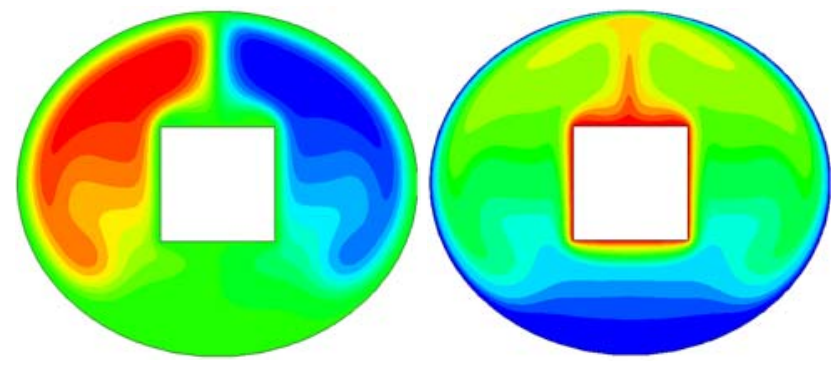

c) $\mathrm{GR}=0.6$
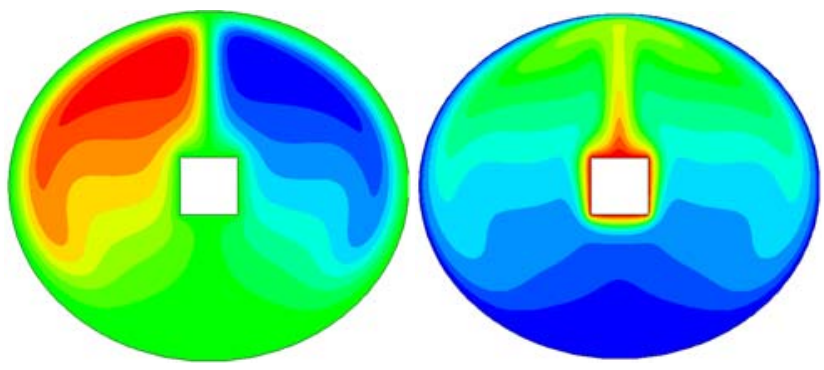

b) $\mathrm{GR}=0.4$
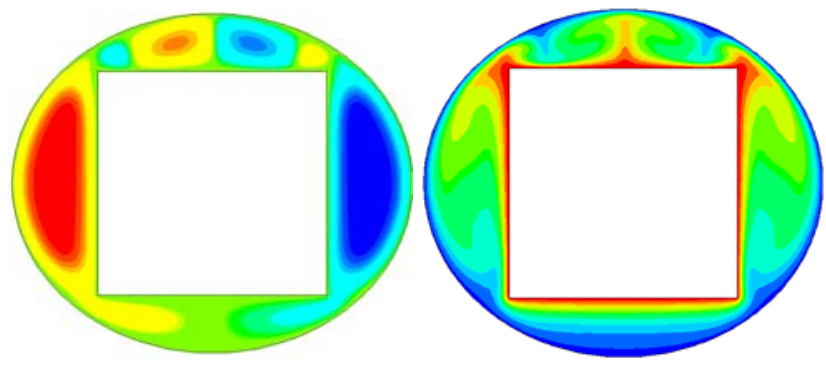

d) $\mathrm{GR}=0.8$

Fig.8. Current lines and isotherm distribution for $\mathrm{Ra}_{\mathrm{t}}=10^{6}$.

\subsection{Effect of Geometry Ratio (GR)}

For the value of the Rayleigh number $\mathrm{Ra}_{\mathrm{t}}=10^{3}$, it can be said that most of the heat transfer is done by conduction at the level of the heated wall. However, the value of the Geometry Ratio GR, for $\mathrm{Ra}_{\mathrm{t}}=10^{4}$, when GR $=0.8$, the free space between the two exchange surfaces is small. This fact promotes the conductive transfer with the decrease of the Geometry Ratio GR. This free space expands to favor the 
convective transfer which remains relatively low. For $\mathrm{Ra}_{\mathrm{t}}=10^{5}$ and $\mathrm{Ra}_{\mathrm{t}}=10^{6}$, the heat transfer rate and the value of the current function are higher. Then, it has been noted that when the thermal Rayleigh number increases, the rate of heat transfer also increases. By increasing the value of the Geometry Ratio GR, there is a reduction in the free space between the two exchange surfaces. This leads to an intensification of natural convection. The regime of the flow remains single-celled for a Rayleigh number $\mathrm{Ra}_{\mathrm{t}}=10^{3}$ and $\mathrm{Ra}_{\mathrm{t}}=10^{4}$ regardless of the value of GR. The flow regime becomes multicellular when $\mathrm{Ra}_{\mathrm{t}} \geq 10^{5}$ and $\mathrm{GR}=0.8$. These results clearly illustrate the bifurcations with the four additional cells each rotating in the opposite direction of its neighboring cell. The increase of the Geometry Ratio GR allowed switching to another flow regime which is a multicellular flow.

\subsection{Local Nusselt number on the elliptical outer wall}

Figure 9 illustrates the variation of the local Nusselt number on the outer elliptical wall. This variation confirms that the increase in the thermal Rayleigh number generates an intensification of natural convection which implies an increase in the values of the local Nusselt numbers. For GR $=0.2, \mathrm{GR}=0.4$ and GR $=0.6$, the variation of the local Nusselt number shows the existence of maximum values at the position $\alpha=90^{\circ}$ in the upper half of the annular space. This is due to the particles of the fluid moving upwards under the action of buoyancy forces of Archimedes. The juxtaposition of two counter-rotating cells at this position brings the fluid to the cylindrical wall. A minimum value appears in the lower half of the annular space between $240^{\circ} \leq \alpha \leq 310^{\circ}$ However, for GR $=0.8$ the variation of the local Nusselt number shows the existence of three maxima and two minima in the upper part of the annular space $\left(45^{\circ}<\alpha<135^{\circ}\right)$ due to the transition of a bicellular regime towards a multicellular regime. This fact confirms the appearance of four counter-rotating cells which bring the fluid to the cylindrical wall (case of the maxima) and move away from it (case of the minima).
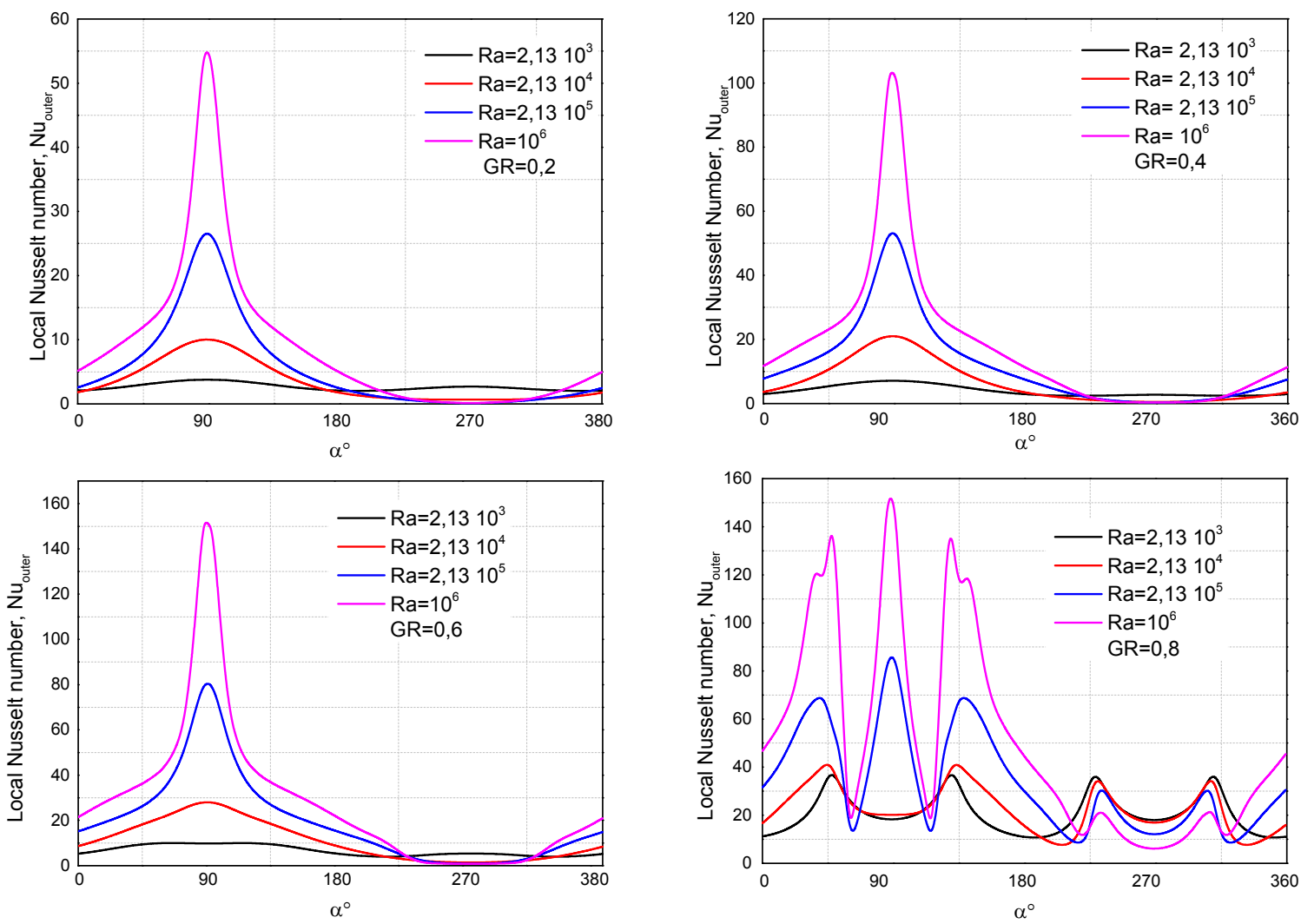

Fig.9. Variation of the number of local Nusselt on the outer wall. 


\subsection{Local Nusselt number on the square inner wall}

Figure10 illustrates the variation of the local Nusselt number as a function of the thermal Rayleigh number. We always notice that the increase of the thermal Rayleigh number causes an intensification of natural convection which implies an increase in the values of the local Nusselt numbers, which is obvious. Convective transfer intensifies and the rate of exchange between the walls and the fluid increases. For GR = $0.2, \mathrm{GR}=0.4$ and $\mathrm{GR}=0.6$, the number of local Nusselt is greater on the corners of the square tube. In fact, it has been observed that the local Nusselt number is larger at the two lower corners than at the two upper corners The curves have only one minimum in the middle of the upper side of the square for GR $\neq 0.8$. Whereas for GR $=0.8$ the curves have three minima and two maxima on the upper side of the square. This fact is due to bifurcations which give rise to four cells each turning in the opposite direction of its neighboring cell. These pairs of cells bring the fluid to the upper wall of the square (case of maxima) and move it away from it (case of the minima).
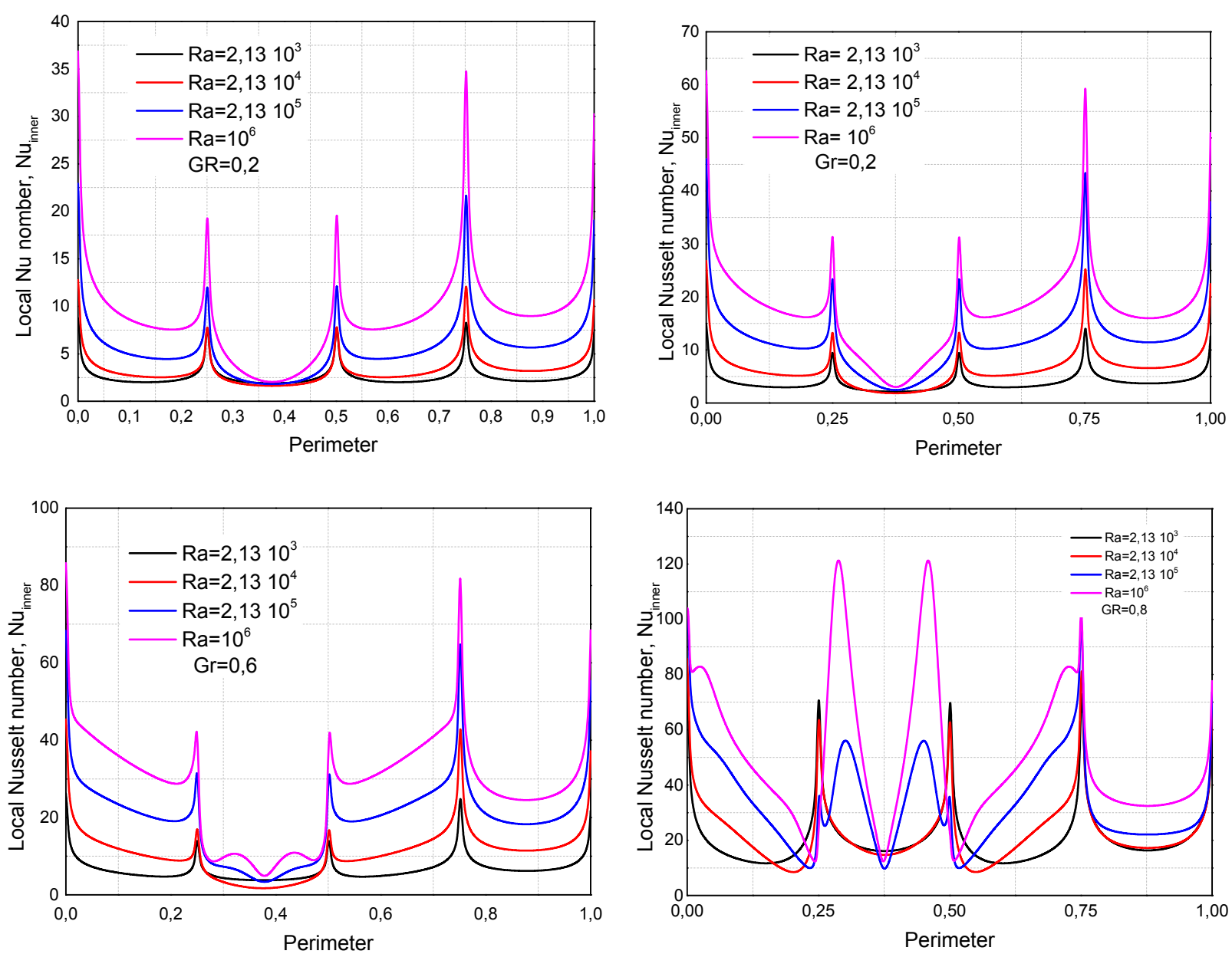

Fig.10. Variation of the local Nusselt number on the inner wall.

\subsection{Variation of the average Nusselt number as a function of the Rayleigh number}

Figure 11 illustrates well the variation of the average Nusselt number as a function of the thermal Rayleigh number for different values of the Geometry Ratio GR. From these results it can be seen that the average Nusselt number increases with increasing the Geometry Ratio. Also, it increases with increasing of 
Rayleigh number. This means that the rate of heat transfer increases with increasing the aspect ratio and thermal Rayleigh number.

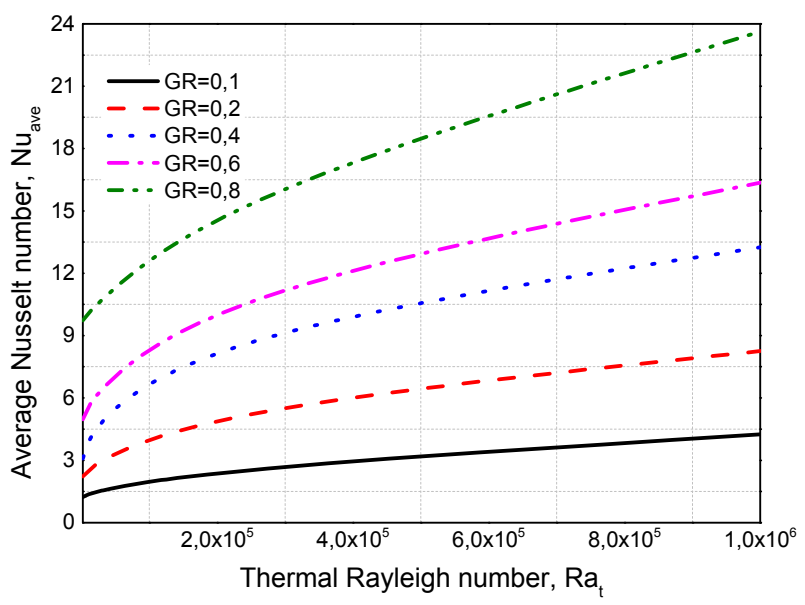

Fig.11. Variation of the average Nusselt number.

\section{Conclusion}

The effects of geometry ratios GR and Rayleigh number on Nusselt numbers, current lines, and isotherms are studied numerically to characterize the natural convective flow in a horizontal annular space between a heated and square inner cylinder and a cold elliptical outer cylinder.

From this study; we can draw the following conclusions:

- For $\mathrm{Ra}_{\mathrm{t}}=10^{3}$, the laminar convection is weak, the values of the current function are very low and almost similar. So, the heat transfer is mainly by conduction.

- For $\mathrm{Ra}_{\mathrm{t}}=10^{5}$ and $\mathrm{Ra}_{\mathrm{t}}=10^{6}$, the increase of the Rayleigh number results in an intensification of natural convection.

- When the Geometry Ratio is equal to $\mathrm{GR}=0.8$, the free space between the two exchange surfaces is small and it favors the conductive transfer. However, with the decrease of the geometry ratio $\mathrm{B}$, the free space will expand to favor the convective transfer that remains relatively low.

- The flux regime remains single-celled for a Rayleigh number $\mathrm{Ra}_{\mathrm{t}}=10^{3}$ and $\mathrm{Ra}_{\mathrm{t}}=10^{4}$ irrespective of the value of the Geometry Ratio GR. However, the flow regime becomes multicellular when $\mathrm{Ra}_{\mathrm{t}} \geq 10^{5}$ and $\mathrm{GR}=0.8$.

- The average Nusselt number increases with the increase of the geometry ratio GR.

In the future, it is interesting to study the effects of the other main controlling parameters, such as the inclination of the enclosure and the Prandtl number (Pr) on the flow structure and heat transfer.

\section{Nomenclature}

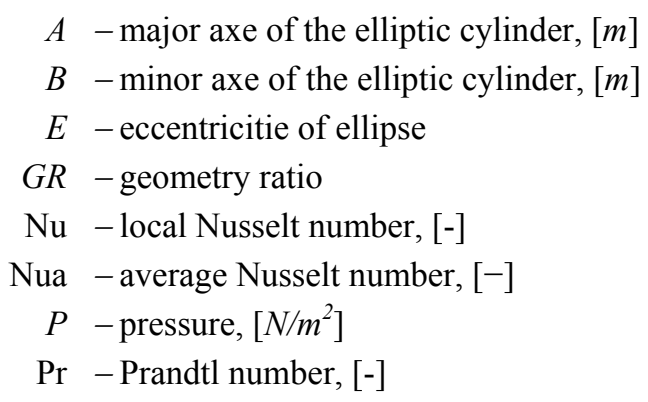




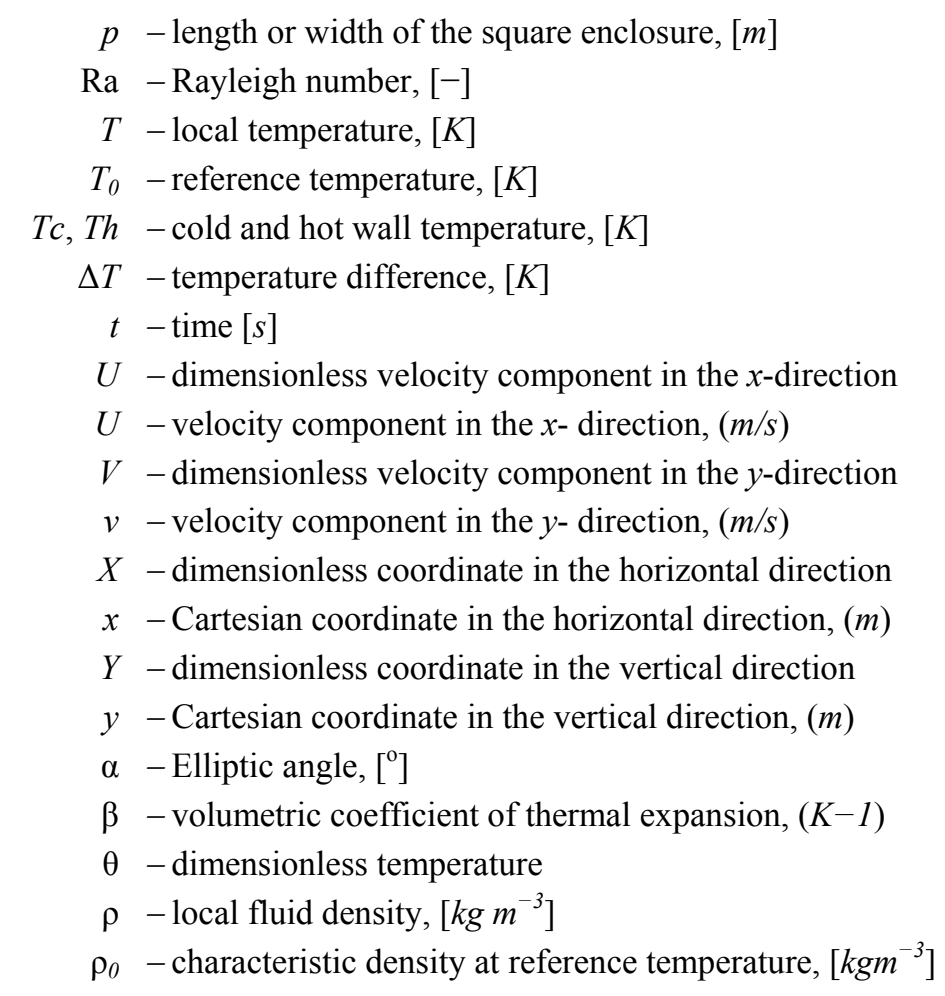

\section{References}

[1] Kuehn T.H. and Goldstein R.J. (1978): An experimental study of natural convection heat transfer in concentric and eccentric horizontal cylindrical annuli. - J. Heat Transfer, vol.100. No.4, pp.635-640.

[2] Kuehn T.H. and Goldstein R.J. (1980): A parametric study of prandtl number and diameter ratio effects on natural convection heat transfer in horizontal cylindrical annuli. - J. Heat Transfer, vol.102, pp.768-770.

[3] Manal H. Al-HafidhRaed G. Saihood (2008): Parametric study of mixed convective radiative heat transfer in an inclined annulus. - Al-Khwarizmi Engineering Journal, vol.4, No.4, pp.45-56.

[4] Lacroix M. and Joyeux A. (1996): Coupling of wall conduction with natural convection from heated cylinders in a rectangular enclosure. - International Communication of Heat and Mass Transfer, vol.23, pp.143-151.

[5] Mohammed Abed W., Shareef A.J. and Najeeb A.A. (2010): Annulus between outer cylinder and inner flat tube. Anbar Journal for Engineering Sciences, AJES, vol.3, No.2.

[6] Lee J.H., Back Y.R., Lee S.R. and Faghri M. (1992): Natural convection in enclosures with an irregular wall. - In: Reizes, J.A. (Ed.), Transport Phenomena in Heat and Mass Transfer. Elsevier, pp.112-123.

[7] Cheng C.H. and Chao C.C. (1996): Numerical prediction of the buoyancy-driven flow in the annulus between horizontal eccentric elliptical cylinders. - Numerical Heat Transfer Part A 30, pp.283-303.

[8] Bouras A., Djezzar M., Naji H. and Ghernoug C. (2014): Numerical computation of double-diffusive natural convective flow within an elliptic-shape enclosure. - International Communications in Heat and Mass Transfer, vol.57, pp.183-192.

[9] Bouras A., Djezzar M. and Ghernoug C. (2013): Numerical simulation of natural convection between two elliptical cylinders: Influence of Rayleigh number and Prandtl number. - Energy Procedia, vol.36, pp.788-797.

[10] Li H. and Tong S. (2016): Natural convective heat transfer in the inclined rectangular cavities with low widthtoheight ratios. - International Journal of Heat and Mass Transfer, vol.93, pp.398-407. 
[11] Awasarmol U.V. and Pise A.T. (2015): An experimental investigation of natural convection heat transfer enhancement from perforated rectangular fins array at different inclinations. - Experimental Thermal and Fluid Science, vol.68, pp.145-154.

[12] El Shamy M.M., Ozisik M.N. and Coulter J.P. (1990): Correlation for laminar natural convection between confocal horizontal elliptical cylinders. - Numer. Heat Transfer, Part A 18, pp.95-112.

[13] Bouras A., Taloub D., Djezza M. and Driss Z. (2018): Natural convective heat transfer from a heated horizontal elliptical cylinder to its coaxial square enclosure.- Mathematical Modelling of Engineering Problems, vol.5, No.4, pp.379-385.

[14] Zhang K., Yang M. and Zhang Y. (2011): Numerical analysis of natural convection in a cylindrical envelope with an internal concentric cylinder with slots, number. - Heat Transfer A, vol.59, No.10, pp.739-754.

[15] Yu Z.T., Hu Y.C., Fan L.W. and Cen K.F. (2010): A parametric study of prandtl number effects on laminar natural convection heat transfer from a horizontal circular cylinder to its coaxial triangular enclosure, number. - Heat Transfer A vol.58, No.7, pp.564-580.

[16] Farooq Hassan Ali Alinnawi Ali Safa Nouri Alsaegh Najlaa Ali Hussein (2013): Laminar natural convection in square enclosure containing different cross sections of inner pipes with internal heat generation. - Journal of Babylon University/ Engineering Sciences / No.2. vol.21.

[17] Mehrizi A.A., Sedighi K., Farhadi M. and Sheikholeslami M. (2013): Lattice Boltzmann simulation of natural convection heat transfer in an elliptical-triangular annulus. - International Communications in Heat and Mass Transfer, vol.48, pp.164-177.

[18] Xing Y., Fatemeh T. and Kambiz V. (2015): Analysis of natural convection in horizontal concentric annuli of varying inner shape numerical heat transfer. - Part A, vol.68, pp.1155-1174.

Received: February 20, 2020

Revised: July 29, 2020 